EXTENDED REPORT

\title{
A newly developed peripheral anterior chamber depth analysis system: principle, accuracy, and reproducibility
}

\author{
K Kashiwagi, F Kashiwagi, Y Toda, K Osada, T Tsumura, S Tsukahara
}

Br J Ophthalmol 2004;88:1030-1035. doi: 10.1136/bjo.2003.036699

See end of article for authors' affiliations

.....................

Correspondence to: Kenii Kashiwagi, $M D$, Department of Ophithalmology, University of Yamanashi Faculty of Medicine, 1110

Shimokato, Tamaho, Yamanashi 409-3898, Japan; keniik@yamanashi. ac.jp

Accepted for publication 7 December 2003

\begin{abstract}
Aim: To develop a new, non-contact system for measuring anterior chamber depth (ACD) quantitatively, and to investigate its accuracy as well as interobserver and intraobserver reproducibility.

Methods: The system scanned the ACD from the optical axis to the limbus in approximately 0.5 second and took 21 consecutive slit lamp images at $0.4 \mathrm{~mm}$ intervals. A computer installed program automatically evaluated the $A C D$, central corneal thickness $(C T)$, and corneal radius of curvature $(C R C)$ instantly. $A$ dummy eye was used for investigating measurement accuracy. The effects of CT and CRC on the measurement results were examined using a computer simulation model to minimise measurement errors. Three examiners measured the $A C D$ in 10 normal eyes, and interobserver and intraobserver reproducibility was analysed.

Results: The ACD values measured by this system were very similar to theoretical values. Increase of CRC and decrease in CT decreased ACD and vice versa. Data calibration using evaluated CT and CRC successfully reduced measurement errors. Intraobserver and interobserver variations were small. Their coefficient variation values were $7.4 \%$ (SD 2.3\%) and $6.7 \%(0.7 \%)$, and these values tended to increase along the distance from the optical axis.

Conclusion: The current system can measure ACD with high accuracy as well as high intraobserver and interobserver reproducibility. It has potential use in measuring $A C D$ quantitatively and screening subjects with narrow angle.
\end{abstract}

$\mathrm{t}$ is important to observe and evaluate the anterior chamber configuration for the diagnosis and treatment of glaucoma. Several methods for evaluating anterior chamber depth (ACD) and configuration have been developed, including ultrasound biomicroscopy (UBM), A-mode ultrasonography, pachymetry, and Scheimpflug principle imaging. ${ }^{1-11}$ Although those methods provide important information for treating patients and understanding the mechanism of angle closure glaucoma (ACG), they present several disadvantages, such as low accuracy, invasiveness, expensiveness, low reproducibility, handling difficulty, and time consuming analysis, which interfere with their widespread use in routine clinical practice or glaucoma screening.

We have developed a new optical system for measuring ACD quantitatively, which can scan the ACD from the optical axis to the limbus consecutively without invasion. In this study, we demonstrate the principle, configuration, accuracy, and intraobserver and interobserver reproducibility of this system in order to evaluate its usefulness in routine clinical procedure or glaucoma screening practice.

\section{METHODS}

The portion of the study that involved the use of human eyes was approved by the Yamanashi University ethics committee, and informed consent was obtained from all the participants based on the Helsinki Declaration.

\section{System configuration}

The system was named scanning peripheral anterior chamber depth analyser, or SPAC, and its configuration is shown in figure 1. The system was composed of a photographic unit and an analytical unit. The photographic unit comprised a slit lamp microscope (Takagi SM70, Takagi Seiko, Nagano, Japan) and a $1 / 3$ inch charge coupled device (CCD) camera (Sentech Sensor Technology, STC-160CS, 380,000 pixels, Kanagawa, Japan). The analytical unit was composed of a personal computer Model MA20V/B (NEC, Tokyo, Japan) installed with a graphics board FDM-PCI-2 (Photoron, Tokyo, Japan) for image analysis, a 14 inch display, and a printer. Image data was input using a National Television Standard Committee (NTSC) system, and the resolution was $640 \times 480$ pixels. Windows 2000 Professional (Microsoft, Redmond, WA, USA) was used as the operating system of the personal computer, and the program was created originally with Watcom C++ (SciTech Software, Chico, CA, USA).

\section{Default setting for measurement}

The default settings were as follows: corneal refractive index, 1.376; anterior chamber refractive index, 1.336; corneal anterior radius of curvature, $7.8 \mathrm{~mm}$; corneal posterior radius of curvature, $6.8 \mathrm{~mm}$; central corneal thickness, $0.5 \mathrm{~mm}$; and peripheral corneal thickness, $0.8 \mathrm{~mm}$. The light used in photography was irradiated from the temporal side at an angle of 60 degrees against the optic axis, the slit had a total length of $5 \mathrm{~mm}$ and a width of $0.1 \mathrm{~mm}$, and the slit lamp was linearly moved from the pupil centre towards the peripheral contour at a rate of $12 \mathrm{~mm} / \mathrm{s}$. An $8 \mathrm{~mm}$ scan required 0.667 second. The optics for slit cross sectional photography employed a photographic magnification factor of 0.4 , and a total of 21 images were photographed consecutively at $0.4 \mathrm{~mm}$ or $33 \mathrm{~ms}$ intervals.

\section{Photography methods}

Similar to normal slit lamp examination, the subject was instructed to set his/her head against the forehead and chin rest of the slit lamp and then gaze at a red light emitting diode (LED) in front for fixation and alignment. All

Abbreviations: $A C D$, anterior chamber depth; $C R C$, corneal radius of curvature; $C T$, corneal thickness; LED, light emitting diode; PACD, peripheral anterior chamber depth; SPAC, scanning peripheral anterior chamber depth analyser; UBM, ultrasound biomicroscopy 


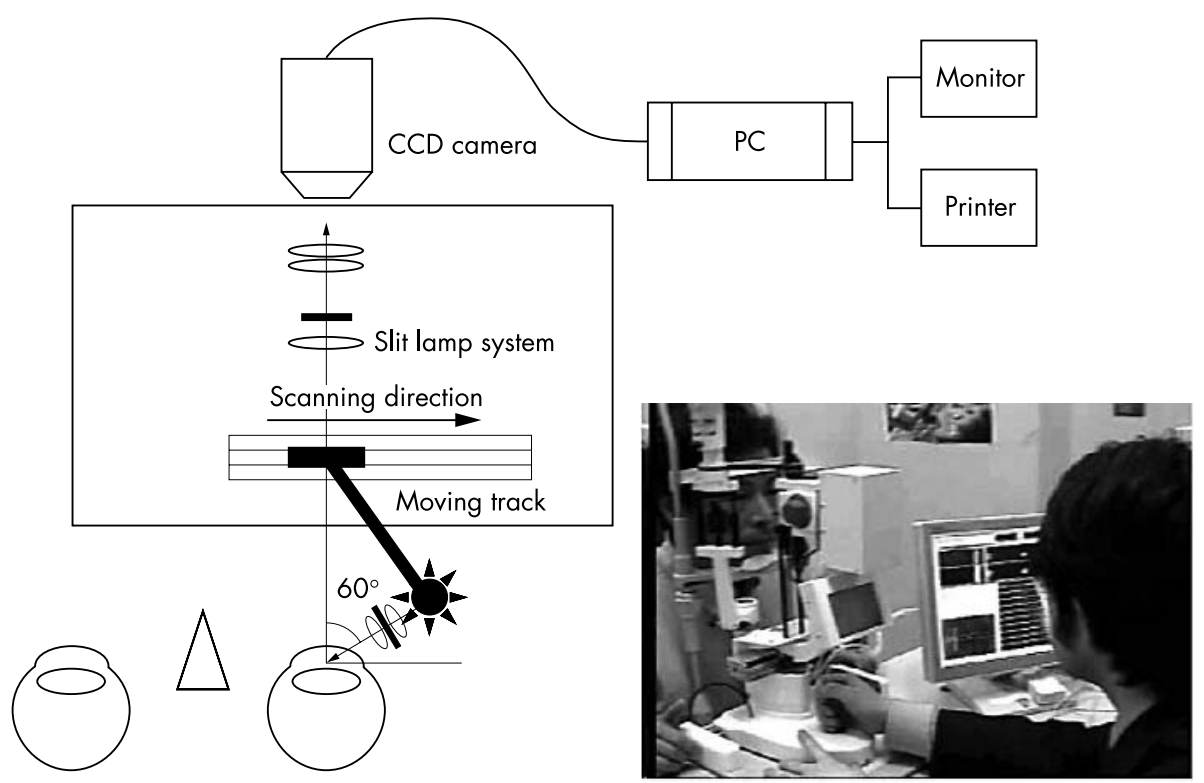

Figure 1 Configuration of the SPAC system and the photograph of the SPAC equipment. The system is composed of a slit lamp microscope, a CCD camera, a computer, and a printer. The patient is instructed to gaze into a fixation lamp. The light from the slit lamp is illuminated towards the subject's eye at an angle of 60 degrees from the temporal side, and moved along a track in the temporal direction. Twenty one consecutive slit lamp images are captured with the CCD camera, the image data are instantaneously analysed by the computer, and the results are printed out by the printer. $C C D=$ charged coupled device, $P C=$ personal computer. The photograph of the SPAC equipment is shown at the bottom right.

parameters including the illumination were controlled throughout the study. The examiner then aligned the reflection of the alignment LED with the pupil centre. Next, focus was adjusted to superimpose the slit lamp light and the reflected mirror image of the slit lamp light on the cornea. When the focus was aligned, images were photographed by pressing a trigger button on the joystick lever of the slit lamp. Photography of a single measurement was completed in 0.667 second, which is the time required for completing an $8 \mathrm{~mm}$ scan. The NTSC formatted video signals of 21 consecutively photographed images from the CCD camera were input to the graphics board, and the processed image data were immediately analysed by an image analysis program. The analysed results and the photographed images were immediately shown on the display. Images judged to be imperfect were excluded from the analysis in the cases indicated in table 1.

\section{Image analysis program and displayed images}

Consecutively photographed images with a monitor screen and ACD values (fig 2A), a single arbitrarily photographed image (fig 2B), a plot of the determined values of ACD (fig 2C), and a cross sectional image of the anterior ocular segment (fig 2D) are shown. The margins of the cornea and the iris were determined from the photographed images as follows. One photographed image (fig 3A) was represented by a histogram in which the brightness of 100 vertical pixels

\section{Table 1 Exclusion criteria}

1 When fixation was judged to be improper because the alignment fixation lamp could not be observed at the centre of the cornea 2 When focus was judged to be improper as a result of failure to superimpose the slit lamp light and the reflected image of the fixation indicator

3 When consecutive images could not be photographed because the subject blinked or moved during photography

4 When slit light observation was inadequate because of improper eyelid opening resulting in the photography of eyelashes and so forth 5 When it was difficult to identify the anterior surface of the iris with the slit lamp microscope because of extreme mydriasis

6 When it was difficult to adequately observe the anterior chamber with the slit lamp microscope because of low corneal transparency

7 When there were excessive irregularities on the cornea surface such as those following corneal transplant was plotted on the $\mathrm{Y}$ axis (fig 3B). An installed program automatically detects peaks attributed to the reflection of the cornea or the sclera and the iris (fig 3C). The margins determined by this program agreed well with those of the cornea and the iris measured from photographed images (fig 3D). ACD was calculated by taking the average of 100 data of the distance between the cornea and the iris calculated for each section.

\section{Estimation of corneal radius of curvature (CRC)}

The corneal radius of curvature $\mathrm{R}$ was calculated as follows using data from two photographed images-namely, an image focusing on the corneal peak on the optical axis and an image moving at a distance $\mathrm{D}$ towards the posterior side along the optical axis, as shown in figure 4 .

$$
\mathrm{R}=((\mathrm{D}-\mathrm{h} / \tan \theta) 2+\mathrm{h} 2) / 2(\mathrm{D}-\mathrm{h} / \tan \theta)
$$

Here, $\mathrm{h}$ is the distance along the vertical line perpendicular to the optical axis between two corneal epithelial points, a corneal peak on the optical axis, and a corneal refracting point with movement at a distance $\mathrm{D}$ along the optical axis, and $\theta$ is an assumptive angle between the optical axis and the illuminating slit lamp ray $\mathrm{b}$ without refraction by the cornea.

\section{Estimation of corneal thickness (CT)}

Calibrated corneal thickness $\mathrm{Ch}$ along the optical axis was determined using the following equation.

$$
\mathrm{Ch}=0.9733 \times \mathrm{Cm} \cos (30.874)+0.0067
$$

Here, $\mathrm{Cm}$ is measured CT from an image focusing on the corneal peak on the optical axis.

\section{Comparison between SPAC measured ACD values and} theoretical $A C D$ values using a dummy eye (fig 5)

A glass dummy eye having an anterior radius of curvature of $7.8 \mathrm{~mm}$, a diameter of $12 \mathrm{~mm}$, a thickness of $5 \mathrm{~mm}$, and a refractive index of 1.51825 was measured using this system, and the accuracy of the system was assessed by comparing theoretical values with measured values of the dummy eye. The procedure consisted of measuring the immobilised dummy eye from the left and the right. The above measurements were conducted three times and then the 
A
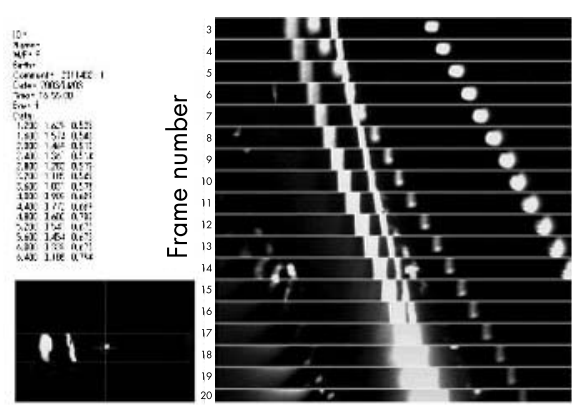

C

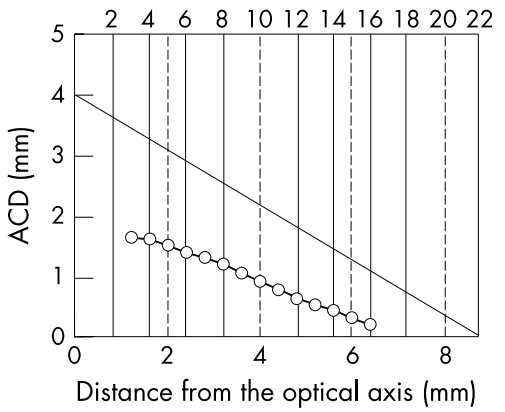

B

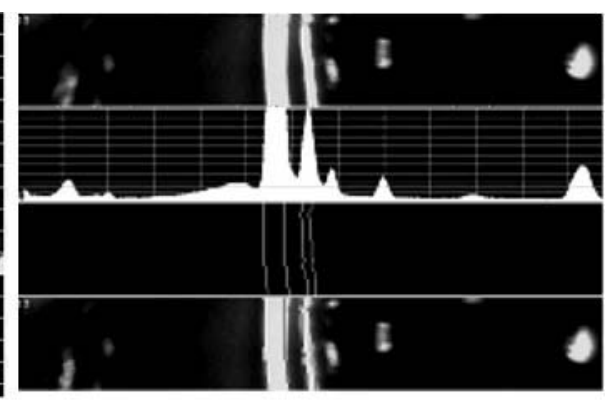

D

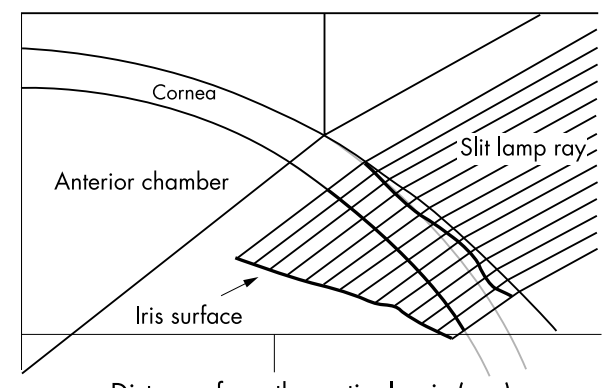

Distance from the optical axis $(\mathrm{mm})$
Figure 2 Determination of margins of cornea and iris. Representative SPAC images of a female with a narrow angle. (A) Twenty one consecutive images (right panel), eye monitoring window (lower left panel), and values (upper left panel) are displayed. (B) Sample image for determining PACD and corneal thickness. A slit lamp image (first row) and a histogram of the number of white pixels calculated from the slit lamp image (second row). Two major peaks indicate cornea (leff) and iris (right). Determined margins of the cornea and the iris (third row). Margins are superimposed on the slit lamp image (fourth row). (C) An installed program creates a plot of PACD profile. (D) Drawing of the anterior ocular segment. Bold lines indicate boundaries of corneal epithelium, corneal endothelium, and iris surface. average value was determined. SPAC measured ACD Lx at position L was compared with theoretically determined ACD $\mathrm{Lx}$ at the same position $\mathrm{L}$.

\section{Assessment of SPAC estimated CRC and CT}

Ten eyes from five healthy subjects (age 26-41 years) were employed to determine the accuracy of SPAC estimated CRC

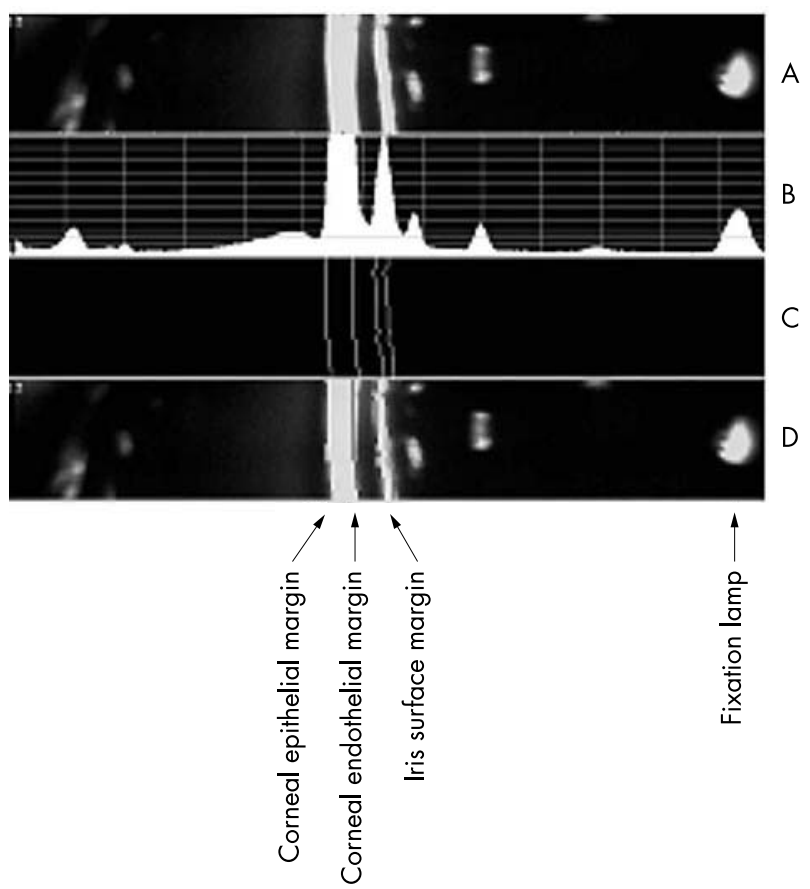

Figure 3 Determination of $A C D$. A photographed image $(A)$ is converted to a histogram (B) and an installed program automatically determined margins of corneal epithelium, corneal endothelium, and iris surface (C). A superimposed image shows that the determined margins agree well with the margins of the cornea and the iris shown on the original images (D). and CT. One experienced ophthalmologist (KK) measured CRC and CT of the enrolled subjects while masking any information regarding SPAC measurement using an automated keratometer (ARK-900, Nidek, Aichi, Japan) and a pachymeter (UP-2000, Nidek), and the values of CRC and CT were compared with those estimated by SPAC using the above described equations 1 and 2 .

\section{Effects of CRC and CT on ACD}

A study was conducted to assess the effects of CRC and CT on the measurement results. As there were few detailed reports of the shape of the anterior surface of the iris of the human eye, the standard shapes of the anterior surface of the cornea and the iris of the human eye were determined based on the

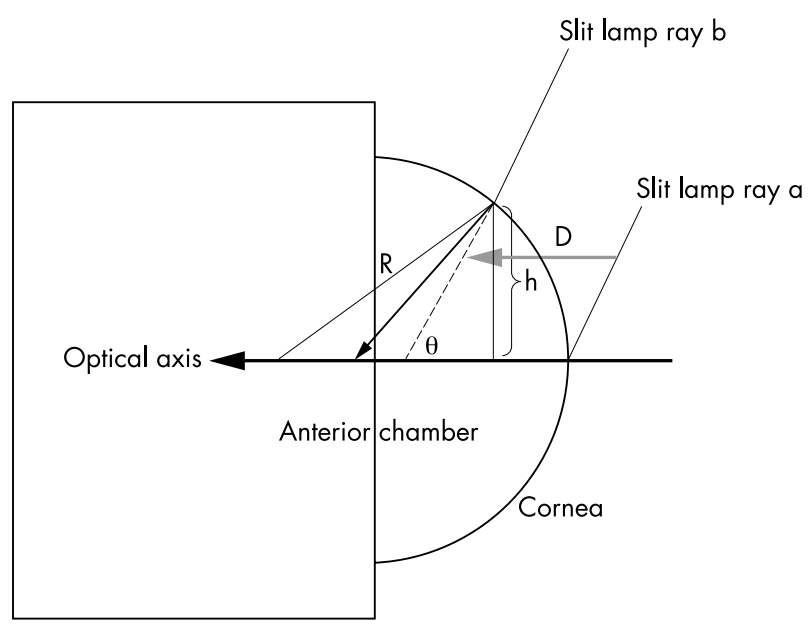

Figure 4 Estimation of corneal radius of curvature. Slit lamp ray a: image taken while focusing on the corneal peak on the optical axis; slit lamp ray b: image taken while moving at a distance $D$ towards the posterior along the optical axis; $\theta$ : an assumptive angle between the optical axis and the illuminating slit lamp light ray without refraction by the cornea; $h$ : scanning distance between image a and image $b$; $R$ : corneal radius of curvature. 


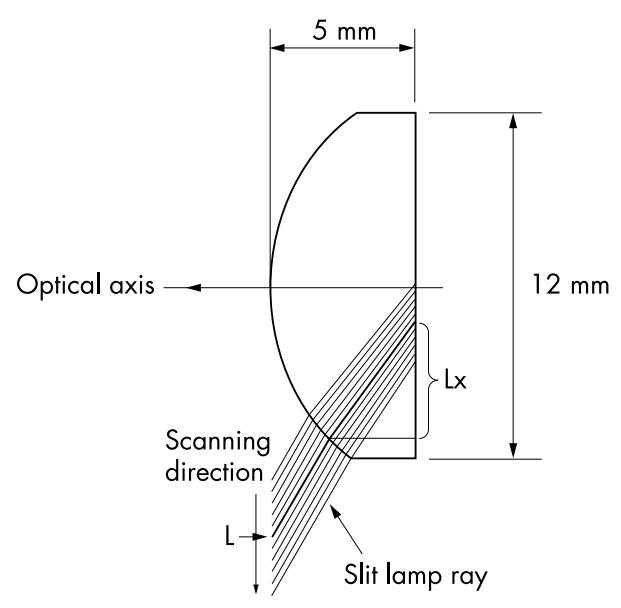

Figure 5 Structure of dummy eye and ACD measurement: $L x$ is SPAC measured $A C D$ at a position $L$.

data obtained by SPAC. The subjects were outpatients having vision of 20/30 or better with an equivalent refractive error of plus or minus 5 dioptres and were free of any abnormal findings in the anterior ocular segment, including the cornea. After excluding subjects exhibiting ACG or pseudophakia, and those who underwent intraocular laser treatment, measurements were performed on 210 eyes of 105 Japanese adults. The subjects consisted of 50 men and 55 women (average age 50.5 (SD 12.3) years). The standard shapes of the corneal anterior surface and that of the iris surface were determined from mean SPAC measured values with theoretically calculated supplemental data using a computer simulation soft, Auto CAD LT 2000 (San Rafael, CA, USA). Simulated equations to draw the above determined standard shape of the corneal anterior surface and that of the iris surface were formulated according to Zernike polynomials that represent wave surface using polar coordinates. ${ }^{12}{ }^{13} \mathrm{~A}$ lens system design software, Code V version 9.20 (Optical Research Associates, Pasadena, CA, USA), automatically simulated changes in ACD when CRC was changed from $7.0 \mathrm{~mm}$ to $8.6 \mathrm{~mm}$ in $0.4 \mathrm{~mm}$ increments or when the central CT was changed from $0.4 \mathrm{~mm}$ to $0.6 \mathrm{~mm}$ in $0.02 \mathrm{~mm}$ increments.

\section{Intraobserver and interobserver measurement error} Ten randomly selected eyes from 10 normal subjects (age 4l60 years) with normal eyes having angle openness classified as Shaffer's grade 3 or 4 , no ocular disease, corrected visual acuity of $20 / 20$ or better, and refractive error within plus or minus 4 dioptres, were used. Measurements were performed three times on each subject's eye by three examiners (two ophthalmologists, $\mathrm{A}$ and $\mathrm{B}$, and one member of the healthcare staff, C) who are familiar with the operation of the system. Then, a comparative study was conducted between intraobserver and interobserver average measured values and coefficients of variation $(\mathrm{CV})$.

\section{Statistical analysis}

The Mann-Whitney U test, and paired $t$ test were employed. Values less than 0.05 were considered to be statistically significant. All data were expressed as means (SD).

\section{RESULTS}

Comparison between SPAC measured ACD values and theoretical $A C D$ values using dummy eye

The relation between the results of SPAC measurement of a dummy eye and the theoretical values is shown in table 2 . The mean difference between SPAC measured ACD values and theoretical ACD values was $0.74 \%(0.51 \%)$, with the range being from $0.009 \%$ to $1.772 \%$.

\section{Accuracy of SPAC measured CT and CRC}

CT values estimated either by SPAC or the pachymeter were $0.504(0.026) \mathrm{mm}$ or $0.502(0.007) \mathrm{mm}$, respectively. The difference between two measurements is $4.45 \%$ (2.25\%) with the range being from $0.39 \%$ to $7.91 \%$ and there is no significant difference between these two results $(p=0.870$, paired $t$ test). CRC values estimated by SPAC or the pachymeter were $7.71(0.92) \mathrm{mm}$ or $7.75(0.20) \mathrm{mm}$, respectively. The difference between two measurements is $2.10 \%(1.10 \%)$ with the range being from $0.57 \%$ to $3.81 \%$ and there is also no significant difference between two results $(\mathrm{p}=0.895$, paired $t$ test $)$.

\section{Effect of CRC and CT on SPAC measurement}

The effect of CRC on the measured values is shown in table 3. It was found that ACD increased as CRC decreased. The maximum difference in ACD among CRCs ranging from $7.0 \mathrm{~mm}$ to $8.6 \mathrm{~mm}$ was $0.167 \mathrm{~mm}$ and the percentage difference increased with an increase in scanning distance.

The effect of CT on the measured values is shown in table 4. It was found that the ACD increased as the CT increased. The maximum difference in ACD among CTs ranging from $0.40 \mathrm{~mm}$ to $0.60 \mathrm{~mm}$ was $0.212 \mathrm{~mm}$ and the percentage difference increased with an increase in scanning distance.

Table 2 Comparison between SPAC measured $A C D$ values and theoretical $A C D$ values using a dummy eye

\begin{tabular}{lllll}
\hline Scanning distance $(\mathrm{mm})$ & SPAC measured ACD $(\mathbf{m m})$ & Theoretical ACD $(\mathbf{m m})$ & Difference $(\mathbf{m m})$ & Difference $(\%)$ \\
\hline 0.0 & 5.033 & 5.088 & 0.054 & 1.080 \\
0.4 & 4.989 & 5.006 & 0.016 & 0.325 \\
0.8 & 4.920 & 4.915 & 0.005 & 0.094 \\
1.2 & 4.842 & 4.816 & 0.026 & 0.538 \\
1.6 & 4.754 & 4.709 & 0.044 & 0.928 \\
2.0 & 4.604 & 4.594 & 0.010 & 0.210 \\
2.4 & 4.471 & 4.471 & 0.001 & 0.009 \\
2.8 & 4.313 & 4.340 & 0.027 & 0.827 \\
3.2 & 4.166 & 4.200 & 0.034 & 0.989 \\
3.6 & 4.012 & 4.052 & 0.040 & 1.772 \\
4.0 & 3.828 & 3.730 & 0.068 & 0.355 \\
4.4 & 3.763 & 3.557 & 0.057 & 1.016 \\
4.8 & 3.545 & 1.685 & 0.012 & 0.792 \\
5.2 & 1.668 & 1.594 & 0.017 & \\
5.6 & 1.582 & 0.013 & \\
\hline ACD $=$ anterior chamber depth. & & & \\
\hline
\end{tabular}


Table 3 Effect of corneal radius of curvature on SPAC measurement

\begin{tabular}{|c|c|c|c|c|c|c|c|}
\hline \multirow{2}{*}{$\begin{array}{l}\text { Scanning } \\
\text { distance }(\mathrm{mm})\end{array}$} & \multicolumn{5}{|c|}{ Corneal radius of curvature $(\mathrm{mm})$} & \multicolumn{2}{|c|}{ Maximum difference } \\
\hline & 7.0 & 7.4 & 7.8 & 8.2 & 8.6 & $\mathrm{~mm}$ & $\%$ \\
\hline 0.000 & 2.659 & 2.618 & 2.580 & 2.550 & 2.514 & 0.146 & 5.480 \\
\hline 0.400 & 2.528 & 2.488 & 2.451 & 2.421 & 2.386 & 0.142 & 5.624 \\
\hline 0.800 & 2.398 & 2.360 & 2.324 & 2.294 & 2.261 & 0.138 & 5.742 \\
\hline 1.200 & 2.270 & 2.233 & 2.199 & 2.170 & 2.137 & 0.133 & 5.868 \\
\hline 1.600 & 2.143 & 2.107 & 2.074 & 2.045 & 2.013 & 0.129 & 6.040 \\
\hline 2.000 & 2.015 & 1.980 & 1.948 & 1.919 & 1.888 & 0.127 & 6.300 \\
\hline 2.400 & 1.884 & 1.850 & 1.818 & 1.788 & 1.757 & 0.126 & 6.704 \\
\hline 2.800 & 1.746 & 1.713 & 1.680 & 1.649 & 1.618 & 0.128 & 7.323 \\
\hline 3.200 & 1.599 & 1.565 & 1.532 & 1.499 & 1.467 & 0.132 & 8.263 \\
\hline 3.600 & 1.438 & 1.403 & 1.368 & 1.332 & 1.298 & 0.140 & 9.703 \\
\hline 4.000 & 1.256 & 1.219 & 1.182 & 1.142 & 1.106 & 0.150 & 11.930 \\
\hline 4.400 & 1.046 & 1.008 & 0.968 & 0.923 & 0.885 & 0.161 & 15.409 \\
\hline 4.800 & 0.805 & 0.766 & 0.725 & 0.676 & 0.638 & 0.167 & 20.733 \\
\hline 5.200 & 0.539 & 0.504 & 0.466 & 0.420 & NA & 0.120 & 22.206 \\
\hline 5.600 & 0.287 & 0.261 & 0.231 & 0.193 & NA & 0.094 & 32.811 \\
\hline 6.000 & 0.089 & 0.073 & 0.052 & 0.022 & NA & 0.067 & 75.294 \\
\hline
\end{tabular}

\section{Measurement reproducibility}

Intraobserver and interobserver variations were small, and their mean coefficient of variance $(\mathrm{CV})$ values were $7.4 \%$ $(2.3 \%)$ and $6.7 \%(0.7 \%)$. Intraobserver $\mathrm{CV}$ values among three examiners were 8.0\% (3.6\%) (ophthalmologist A), 7.3\% $(2.6 \%)$ (ophthalmologist B), and $6.9 \%$ (1.7\%) (healthcare staff member, C). When comparing the CV among the examiners, there was no difference. Interobserver and intraobserver variations tended to deteriorate along the distance from the optical axis. Figure 6 shows the change in the interobserver $\mathrm{CV}$ in $\mathrm{ACD}$ measurement along the distance from the optical axis.

\section{DISCUSSION}

Previously reported anterior chamber measurement systems can be broadly classified into those using ultrasonic waves (UBM) and those using optics. ${ }^{1-11}$ UBM allows observation of the anterior chamber angle configuration, and its quantitative aspects have improved because of modifications of the measurement program in recent years. ${ }^{14}$ As a result, today UBM is considered to be one of the most reliable methods for the diagnosis of ACG. However, in addition to using contact, the reproducibility of this method is somewhat low and measurement accuracy is affected in particular by the dexterity of the examiner. The use of this method is limited to ophthalmologists. Although optical methods for anterior chamber angle evaluation have also been reported, the values measured by such optical systems are affected by refraction, and the systems are expensive. ${ }^{6710}$ Optical systems have several shortcomings, including the lack of a proper program for quantitative measurement of peripheral anterior chamber depth (PACD), and even if such a program exists, a considerable amount of effort is required for its interpretation. ${ }^{15}$

The recently developed SPAC system can differentiate eyes with narrow angle from those with wide open angle. We investigated the sensitivity and specificity of the SPAC system for detecting eyes with narrow angel from more than 1000

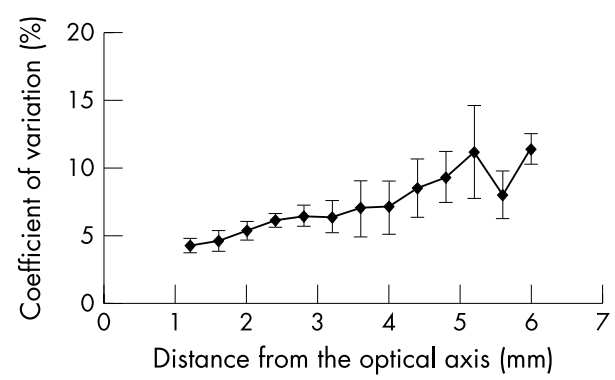

Figure 6 Interobserver coefficient variance in ACD measurement.

Table 4 Effect of corneal thickness on SPAC measurement

\begin{tabular}{|c|c|c|c|c|c|c|c|c|c|c|c|c|c|}
\hline \multirow{2}{*}{$\begin{array}{l}\text { Scanning distance } \\
(\mathrm{mm})\end{array}$} & \multicolumn{11}{|c|}{ Corneal thickness (mm) } & \multicolumn{2}{|c|}{ maximum difference } \\
\hline & 0.40 & 0.42 & 0.44 & 0.46 & 0.48 & 0.50 & 0.52 & 0.54 & 0.56 & 0.58 & 0.60 & $\mathrm{~mm}$ & $\%$ \\
\hline 0.0 & 2.520 & 2.532 & 2.544 & 2.556 & 2.568 & 2.580 & 2.593 & 2.605 & 2.617 & 2.630 & 2.642 & 0.123 & 4.646 \\
\hline 0.4 & 2.392 & 2.404 & 2.415 & 2.427 & 2.439 & 2.451 & 2.463 & 2.475 & 2.487 & 2.499 & 2.511 & 0.120 & 4.758 \\
\hline 0.8 & 2.266 & 2.278 & 2.289 & 2.301 & 2.312 & 2.324 & 2.335 & 2.347 & 2.359 & 2.371 & 2.383 & 0.116 & 4.880 \\
\hline 1.2 & 2.142 & 2.153 & 2.165 & 2.176 & 2.187 & 2.199 & 2.210 & 2.221 & 2.233 & 2.244 & 2.256 & 0.114 & 5.046 \\
\hline 1.6 & 2.018 & 2.029 & 2.040 & 2.051 & 2.063 & 2.074 & 2.085 & 2.096 & 2.108 & 2.119 & 2.130 & 0.113 & 5.297 \\
\hline 2.0 & 1.891 & 1.902 & 1.913 & 1.925 & 1.936 & 1.948 & 1.959 & 1.970 & 1.982 & 1.993 & 2.004 & 0.114 & 5.680 \\
\hline 2.4 & 1.758 & 1.770 & 1.782 & 1.794 & 1.806 & 1.818 & 1.829 & 1.841 & 1.853 & 1.864 & 1.876 & 0.117 & 6.255 \\
\hline 2.8 & 1.618 & 1.630 & 1.643 & 1.655 & 1.668 & 1.680 & 1.693 & 1.705 & 1.717 & 1.729 & 1.741 & 0.124 & 7.112 \\
\hline 3.2 & 1.464 & 1.478 & 1.491 & 1.505 & 1.519 & 1.532 & 1.545 & 1.559 & 1.572 & 1.585 & 1.598 & 0.134 & 8.389 \\
\hline 3.6 & 1.292 & 1.307 & 1.323 & 1.338 & 1.353 & 1.368 & 1.383 & 1.397 & 1.412 & 1.4216 & 1.441 & 0.149 & 10.323 \\
\hline 4.0 & 1.096 & 1.113 & 1.131 & 1.148 & 1.165 & 1.182 & 1.199 & 1.215 & 1.232 & 1.248 & 1.264 & 0.168 & 13.325 \\
\hline 4.4 & 1.870 & 0.890 & 0.910 & 0.929 & 0.949 & 0.968 & 0.987 & 1.006 & 1.025 & 1.044 & 1.062 & 0.192 & 18.087 \\
\hline 4.8 & 0.618 & 0.639 & 0.661 & 0.682 & 0.704 & 0.725 & 0.746 & 0.67 & 0.788 & 0.809 & 0.830 & 0.212 & 25.535 \\
\hline 5.2 & 0.364 & 0.384 & 0.404 & 0.425 & 0.446 & 0.466 & 0.488 & 0.509 & 0.530 & 0.551 & 0.573 & 0.209 & 36.410 \\
\hline 5.6 & 0.151 & 0.166 & 0.182 & 0.198 & 0.214 & 0.231 & 0.249 & 0.267 & 0.285 & 0.303 & 0.322 & 0.172 & 53.265 \\
\hline 6.0 & NA & 0.006 & 0.017 & 0.028 & 0.040 & 0.052 & 0.064 & 0.077 & 0.090 & 0.103 & 0.177 & 0.111 & 94.994 \\
\hline
\end{tabular}


eyes with glaucoma, and it was revealed that the SPAC system has quite good sensitivity and specificity. This investigation also showed that the percentage of eyes that did not provide useful results was less than 3\% in this study (data in preparation).

Because the SPAC system is an optical system, it is completely non-contact and allows quantitative measurement of PACD. In addition, as indicated in the results described above, the SPAC system enables measurement of PACD by examiners who are not ophthalmologists. In addition, owing to its simple configuration, it can be fabricated at a low cost compared with conventional systems. Operation is easy and it takes less than 10 seconds to complete a single examination including positioning, centration, acquisition, and processing of the images. Our simulation showed that the measured values were found to change as a result of changes in CRC and CT. Therefore, we adjusted the measured ACD values using CRC and CT data and this modification resulted in much more accurate values of ACD. We employed normal eyes for investigating the reproducibility of the SPAC system, because it is sometimes difficult to measure the most peripheral measuring points of eyes with narrow angles. However, the reproducibility of ACD of eyes with narrow angle at the central region is quite similar to the current results (unpublished data).

This system presents several limitations as well as precautions that must be observed when evaluating measurement results. As light is irradiated at an angle of 60 degrees from the temporal side, measurement results cannot be directly compared with previously reported values of ACD. The system cannot be used for observations up to the bottom of the anterior chamber angle as can be done with UBM. It is not capable of detecting conditions such as peripheral anterior synechiae. In addition, as only ACD in the outside direction is evaluated, evaluations cannot be made on the other sites. Measurement at peripheral regions is also difficult for patients with prominent peripheral corneal opacity.

In conclusion, this system enables non-contact and quantitative evaluation of PACD and can be operated by health examiners other than ophthalmologists. Thus, it is considered to be useful for ACG screening.

\section{ACKNOWLEDGEMENTS}

The authors thank Mrs Koji Tagawa and Junji Nakagawa for their excellent technical assistance.

\section{Authors' affiliations}

K Kashiwagi, F Kashiwagi, Y Toda, K Osada, T Tsumura, S Tsukahara, Department of Ophthalmology, University of Yamanashi Faculty of Medicine, Tamaho Yamanashi, Japan

Proprietary interest: Japanese patent application No 2003-111322.

\section{REFERENCES}

1 Gazzard G, Friedman DS, Devereux JG, et al. A prospective ultrasound biomicroscopy evaluation of changes in anterior segment morphology after laser iridotomy in Asian eyes. Ophthalmology 2003;110:630-8.

2 Van Herick W, Shaffer RN, Schwartz A. Estimation of width of angle of anterior chamber. Incidence and significance of the narrow angle. Am J Ophthalmol 1969;68:62-9.

3 Jacobs IH. Anterior chamber depth measurement using the split-lamp microscope. Am J Ophthalmol 1979:88:236-8.

4 Lee DA, Brubaker RF, Ilstrup DM. Anterior chamber dimensions in patients with narrow angles and angle-closure glaucoma. Arch Ophthalmol 1984; 102:46-50.

5 Jin JC, Anderson DR. The effect of iridotomy on iris contour. Am J Ophthalmol 1990;110:260-3.

6 Richards DW, Russell SR, Anderson DR. A method for improved biometry of the anterior chamber with a Scheimpflug technique. Invest Ophthalmol Vis Sci 1988;29:1826-35.

7 Dragomirescu V, Hockwin O. Rotating slit image camera Topcon SL 45. New developments for simultaneous image acquisition by photographic and CCDassisted on-line documentation. Ophthalmic Res 1996;28:102-8.

8 Congdon NG, Spaeth GL, Augsburger J, et al. A proposed simple method for measurement in the anterior chamber angle: biometric gonioscopy. Ophthalmology 1999;106:2161-7.

9 Boker T, Sheqem J, Rauwolf $M$, et al. Anterior chamber angle biometry: a comparison of Scheimpflug photography and ultrasound biomicroscopy. Ophthalmic Res 1995;27:104-9.

10 Sasaki K, Sakamoto Y, Shibata T, et al. The multi-purpose camera: a new anterior eye segment analysis system. Ophthalmic Res 1990;22:3-8.

11 Bosem ME, Morsman D, Lusky M, et al. Reproducibility of quantitative anterior chamber angle measurements with Scheimpflug video imaging. J Glaucoma 1992;1:254-7.

12 Malacara D, DeVore SL. Interferogram evaluation and wavefront fitting. Optical shop testing 1992:455-99.

13 Wang JW, Silva DE. Wavefront interpretation with Zernike polynomials. Appl Opt 1980; 19:1510-18.

14 Ishikawa H, Liebmann JM, Ritch R. Quantitative assessment of the anterior segment using ultrasound biomicroscopy. Curr Opin Ophthalmol 2000;11:133-9.

15 Olbert D. Optical problems of anterior chamber depth biometry by scheimpflug photography. Ophthalmic Res 1991;23:342-7. 